\title{
Electromyography Assessment of Forearm Muscles: Towards the Control of Exoskeleton Hand
}

\author{
N. Abas, W. M. Bukhari, M. A. Abas, M. O. Tokhi, Senior Member, IEEE
}

\begin{abstract}
Hand plays an important role in a human's life by offering physical interaction and grasping capabilities. In most stroke cases, the hand is the most vulnerable part of the body that has a high chance of suffering. This has led to the development of a numerous wearable robotic devices such as exoskeleton hands. The exoskeleton hands can provide physical assistance for stroke survivors to regain their abilities in performing basic activities of daily living and to improve their quality of life. The key challenges in developing such a device do not only lie in designing its mechanical but also in designing its controller. In controlling the exoskeleton hand, the principal criterion is to work according to the user's motion intention. It can be done by utilizing the electromyogram (EMG) signals generated by forearm muscles contributed from the movement and/or grasping abilities of the hand. In this paper, electromyography assessment of forearm muscles towards the control of an exoskeleton hand is presented. The EMG signals are collected non-invasively using multi-channel surface EMG sensors. The contractions of the muscles are detected from several forearm (flexion and extensor) muscles and the data is processed through several pattern recognition steps, before being mapped to various pinching/gripping forces and angular joints. The adaptability and learning process is done through a neural network. The experimental results show separable classes of features and significant range of control inputs that represent the inter-relation between forearm EMG signals, various pinching/gripping forces and angular joints for exoskeleton hand control.
\end{abstract}

\section{INTRODUCTION}

The aim of an exoskeleton hand is to assist stroke survivors to regain their hand strength and functionality, and improve their quality of life. In most stroke cases, hands are the most vulnerable part of the body that have a high chance of suffering; $77 \%$ of the stroke survivors experience upper limb weakness which includes limited hand and wrist movement [1]. Stroke is a sudden illness that happens when there is a disruption to the flow of blood to the brain. There are two types of stroke; ischaemic stroke that is caused by a blockage to an artery cutting off the blood supply to the brain,

*Research supported by The University of Sheffield, Universiti Teknikal Malaysia Melaka (UTeM), and Ministry of Higher Education Malaysia.

N. Abas is with the Automatic Control and System Engineering Department, University of Sheffield, S1 3JD UK, on leave from Mechatronics Department, UTeM, Malaysia (Phone: +606-555-2341; email: nabas1@sheffield.ac.uk or norafizahabas@utem.edu.my).

W. M. Bukhari is with the Automatic Control and System Engineering Department, University of Sheffield, S1 3JD UK (e-mail: wmbbinwandaud1@sheffield.ac.uk).

M. A. Abas is a medical officer at University Health Centre. The National University of Malaysia, Selangor, Malaysia (e-mail: drazman@ukm.edu.my).

M.O. Tokhi, is with the School of Engineering, London South Bank University, SE1 0AA London, UK (e-mail: tokhim@1sbu.ac.uk). and haemorrhagic stroke that is caused when a blood vessel bursts within or on the surface of the brain. According to the UK Stroke Association released statistics in January 2017; there are over 1.2 million stroke survivors living in the UK [1]. Some of the most common effects of stroke are physical. The survivors of stroke can experience muscle weakness, paralysis, stiffness, or changes in sensation, usually on one side of the body. These effects may be incorporated with memory problems making it difficult to move some parts of the body, and causing the survivors to struggle in performing activities of daily life [2]. It reduces the survivors' quality of life. Fortunately, there are various approaches to assist the survivors to regain the functionality of their hands, such as by using a robotic device such as an exoskeleton hand.

An exoskeleton is a novel man-machine intelligent system. It is a wearable machine that is powered by a system of motors with corresponding joints and links that allow limb movement, increased strength and endurance. Exoskeletons have been studied since the 1960s for industrial or medical applications. The first whole-body exoskeleton robot; Hardiman, was actuated and was supposed to be driven by a human operator from the inside of the robot [3]. Pons et al. [4] classified exoskeleton robots into three groups; empowering exoskeleton which extends the strength of human beyond its natural ability while maintaining human control of the robot, orthotic exoskeleton whose mechanical structure maps onto the anatomy of the human limb with a purpose to restore lost or weak functions, and prosthetic robot which substitutes the lost limb after amputation. In this research, the development of an orthotic exoskeleton hand that functions as an assistive device for the post-stroke patient with consideration of user motion intention as control input is studied and explored.

Kiguchi et al. [5] suggested that to activate the exoskeleton robot according to the user motion intention, a force sensor based control can be employed for user without limb problem while for those who are not strong enough to move their limb, EMG-based control with the EMG signals from certain muscles can be adopted to activate the system accordingly. Forearm EMG signal is a tiny voltage generated due to movement of each residual muscle within human forearm which contributes to the movement and/or grasping abilities of the hand. This signal in which the user's motion intention is reflected contains much information of the muscle activity and can imply user's intention $30-100 \mathrm{~ms}$ in advance which is crucial in providing real-time information to facilitate accurate control of the exoskeleton hand [6]. In addition, EMG signal offers advantages in terms of control input as compared to that derived through dynamic or kinematic analysis. Commonly, the signals generated from sensors comprise noises from the user and internal device and often introduce a delay for the controller [7]. 
This paper presents the design of orthotic exoskeleton hand and a fundamental study carried out to determine EMG signal behaviours in establishing the inter-relation between forearm EMG signals, various pinching/gripping forces and finger positions for exoskeleton hand control which can enhance the technology in communication between human and machine. The rest of paper is structured as follows: Section II presents system description with explanation of human hand biomechanics and design of the exoskeleton hand. Section III presents the experimental procedure for EMG data collection and data analysis. Section IV presents the pattern recognition procedure. The experimental results are presented and discussed in Section V and the paper is concluded in Section VI.

\section{SYSTEM DESCRIPTION}

\section{A. Hand Biomechanics}

A key aspect in designing exoskeleton hand is to understand the human hand anatomy and biomechanics for ensuring safe operation and effective function of the device. The human hand is highly articulated with various ranges of motion. The hand (Figure 1) has 27 bones divided into three groups: 8 carpal bones which make up the wrist, 5 metacarpal bones as the root of the hand, and 14 phalanges. Two phalanges form the thumb (proximal and distal phalanges). For the rest of the fingers, the phalanges are divided into three intercalated bony segments: distal, middle, and proximal phalanges. The distal phalanx is located at the fingertip, connected to the proximal phalanx that is located at the base of the finger through middle phalanx. The proximal phalanx is also connected to the metacarpal bones in the palm of the hand [3]. The finger joints are metacarpophalangeal (MCP), proximal interphalangeal (PIP), and distal interphalangeal (DIP) joints. The MCP joint of the hand has two degrees of freedom (DOF) (adduction/abduction and flexion/extension) while the PIP and DIP joints each has single DOF (flexion/extension). The PIP and DIP joints are interdependent as the DIP joint is a passive DOF that is driven by the rotation at the PIP. Hence, each natural finger can be considered as a four DOF mechanism with three active joints and one passive joint [8].

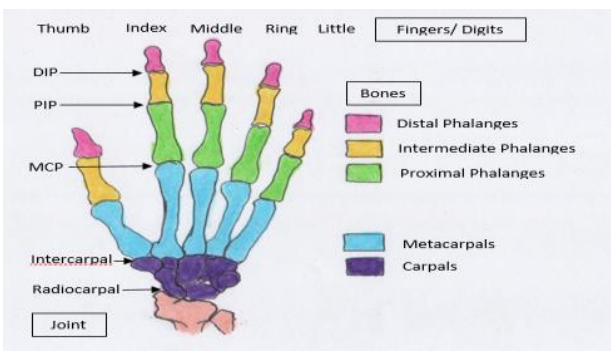

Figure 1. Sketch for bones and joints of a human hand.

\section{B. Exoskeleton Hand}

A considerable amount of literature has been published on the development and design of exoskeleton hand for assistive and rehabilitation purposes. The research developments published for exoskeleton hand are categorized into three; systems assisting wrist movement, systems assisting finger(s) movement, and systems assisting wrist and finger(s) movement. Several researchers have developed systems that support finger(s) movement: assistive [9-13] and rehabilitation [14-16]. Baker et al. [9] developed an orthotic hand assistive exoskeleton (OHAE) that reduces the muscular force needed to pinch or grasp. It has three actuated fingers: the thumb, middle and index fingers, that are driven by cables attached to a glove. Hasegawa et al. [17, 18] have introduced five-fingered assistive hand that supports human hand and wrist activities. It has 11 DOF: eight active joints and three passive joints. A cable-driven mechanism mimicking human finger motion is adopted by the author with each of the joints controlled independently.

In this research, the bony segments of the human hand are closely followed in designing the exoskeleton, with exceptions in the palm and the wrist. The design of index, middle, ring and little fingers contain three intercalated body segments: proximal, middle and distal phalanges, while the thumb contains only two intercalated body segments: proximal and distal phalanges. The physical modeling of the exoskeleton hand is completed in SimMechanics environment. The conceptual design of the exoskeleton hand is done in Solidworks and is imported to the SimMechanics program scheme (Figure 2). This integration allows verification of the model, and facilitates the design process of the controller that is executed in MATLAB environment. Besides, in designing the exoskeleton controller, it is necessary to know dynamics and kinematics of the exoskeleton hand to obtain mathematical models that can closely represent the system. However, the inverse kinematic task is complex to solve analytically and the numerical calculation often entails difficulties as the exoskeleton hand is complex and high in degrees of freedom [19]. With Solidworks and SimMechanics, the mathematical representation of the exoskeleton hand can be developed and modeled without complex mathematical derivations.

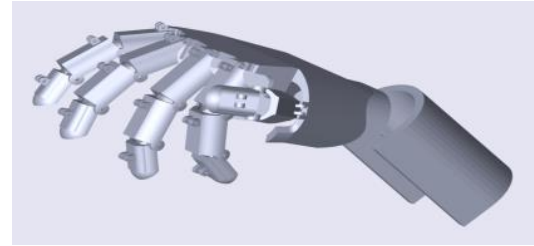

Figure 2. The design of exoskeleton hand in SimMechanics.

In order to develop an accurate and ideal representation of the system, a few assumptions were made to further define the exoskeleton hand model. These are as follows:

1. All the joints (DIP, PIP, and MCP joints) are assumed to have $1 \mathrm{DOF}$, allowing only flexion/extension.

2. All the joint angles (DIP, PIP and MCP joints) for each finger are based on [20] for rectangular object power grip with a grip diameter of $25 \mathrm{~cm}$ (the diameter of hand dynamometer).

3. During the finger pinch, the force is exerted at the middle pulp of distal phalanx and all external forces are assumed to be single unit.

4. All the joint actuators receive forces as an input and render angles as the output (adopting admittance control theory). 


\section{EXPERIMENTAL PROCEDURE}

The EMG datasets utilized in this paper were collected by the authors after obtaining research ethical approval from The University of Sheffield for the purposes of this research. A series of experiments were designed to collect the data. Five subjects were randomly selected; four males and one female aged between 20 - 40 years old. The subjects chosen were normally limbed without any neuro-muscular problems. They were briefed (orally or using a visual aid, ie recorded video) and provided with informed consent prior to the study. The experiment started with experimental set-up, followed by muscles selection and ended with EMG data collection for different finger pinch and hand grip strengths. The muscle excitation and finger pinch force were simultaneously measured using multi-channel EMG sensors and a hand dynamometer by Vernier (HD-BTA). The hand dynamometer is a strain gauge based isometric force sensor that amplifies hand grip force applied to its pressure pads and converts the force value into a corresponding voltage value.

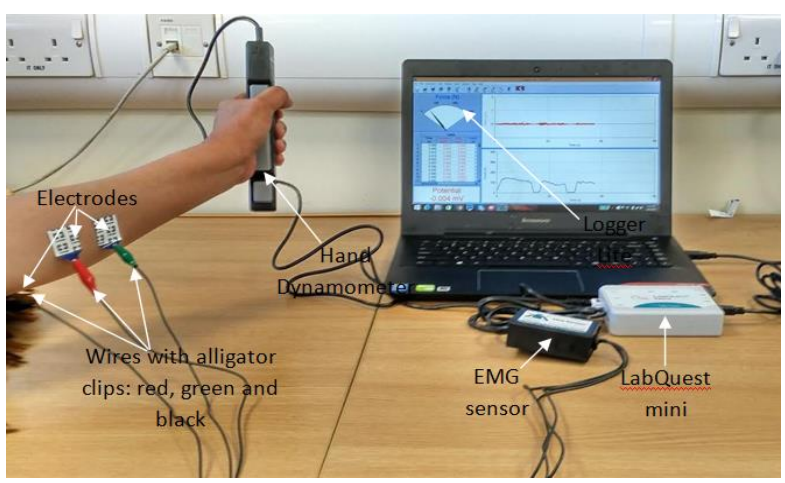

Figure 3. Experimental set-up for EMG and hand grip force measurement.

Figure 3 illustrates the experimental set-up for this research. The areas of the skin where the electrode patches were placed were scrubbed with a paper towel to remove skin oil and moisture. (Detailed skin preparation procedure will only be carried out if necessary). Then, electrode patches were placed on the selected forearm muscle (at the belly of the muscles) on subject's dominant hand with $24 \mathrm{~mm}$ in distance between each electrode and connected to the LabQuest mini data acquisition through the interfacing wire. Next, the hand dynamometer was connected to the LabQuest mini data acquisition and a laptop (battery powered). Finally, Logger Lite software was launched, the sensors and hand dynamometer were calibrated and data were recorded.

\section{A. Muscle Selection}

Combination and coordination actions of both extrinsic and intrinsic musculature have contributed to the dexterous movement of the hand. The extrinsic muscles are originated from the arm and forearm while the intrinsic muscles are located entirely within the hand. Among those muscles, there are several extrinsic muscles that contribute to flexion and extension of the fingers, allocated within forearm posterior and anterior compartments. Assessing the target muscles contributing to the intended finger movement and hand grasping are crucial to characterize the EMG signals classes used in modeling the myoelectric towards the control of exoskeleton hand. The muscles are commonly known as flexor muscles (in the anterior compartment) and extensor muscles (in the posterior compartment) and are separated by the radius and ulna with the interosseous membrane that connects them. Based on the actions intended to be tested [21], five potential muscles (Figure 4 and Figure 5) were selected for further investigation. The list of muscles and their characteristics are listed in Table 1.

TABLE I. MUSCLE UNDER CONSIDERATION WITH ITS CHARACTERISTICS

\begin{tabular}{|c|c|c|c|c|}
\hline No & Muscle & $\begin{array}{c}\text { Distal } \\
\text { Attachment }\end{array}$ & $\begin{array}{l}\text { Forearm } \\
\text { Location }\end{array}$ & Main Actions \\
\hline 1 & $\begin{array}{l}\text { Flexor } \\
\text { Digitorum } \\
\text { Superficialis } \\
\text { (FDS) }\end{array}$ & $\begin{array}{l}\text { Middle } \\
\text { phalanges } \\
\text { of medial } \\
\text { four digits }\end{array}$ & Anterior & $\begin{array}{l}\text { Flexes middle and } \\
\text { proximal phalanges } \\
\text { at PIP and MCP } \\
\text { joints of medial } 4 \\
\text { digits. }\end{array}$ \\
\hline 2 & $\begin{array}{l}\text { Flexor } \\
\text { Digitorum } \\
\text { Profundus } \\
\text { (FDP) }\end{array}$ & $\begin{array}{l}\text { Distal } \\
\text { phalanges } \\
\text { of medial } \\
\text { four digits }\end{array}$ & Anterior & $\begin{array}{l}\text { Flexes distal } \\
\text { phalanges at DIP } \\
\text { joints of medial four } \\
\text { digits; assists the } \\
\text { hand flexion. }\end{array}$ \\
\hline 3 & $\begin{array}{l}\text { Extensor } \\
\text { Digitorum } \\
\text { (ED) }\end{array}$ & $\begin{array}{l}\text { Extensor } \\
\text { expansion } \\
\text { of medial } \\
\text { four digits }\end{array}$ & Posterior & $\begin{array}{l}\text { Extends MCP and } \\
\text { interphalangeal } \\
\text { joints of medial four } \\
\text { digits and extends } \\
\text { hand at wrist joint }\end{array}$ \\
\hline 4 & $\begin{array}{l}\text { Flexor } \\
\text { Pollicis } \\
\text { Longus (FPL) }\end{array}$ & $\begin{array}{l}\text { Distal } \\
\text { phalanx of } \\
\text { first digit }\end{array}$ & Anterior & $\begin{array}{l}\text { Flexes phalanx of } \\
\text { first digit (thumb). }\end{array}$ \\
\hline 5 & $\begin{array}{l}\text { Extensor } \\
\text { Pollicis } \\
\text { Brevis (EPB) }\end{array}$ & $\begin{array}{l}\text { Proximal } \\
\text { phalanx of } \\
\text { first digit }\end{array}$ & Posterior & $\begin{array}{l}\text { Extend proximal } \\
\text { phalanx of first digit } \\
\text { (thumb) at MCP } \\
\text { joint. }\end{array}$ \\
\hline
\end{tabular}

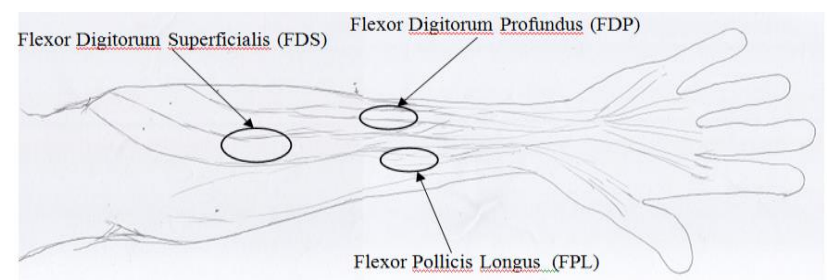

Figure 4. . Sketch for the anterior compartment of a human forearm with the location of muscles under consideration: FDS, FDP, and FPL.

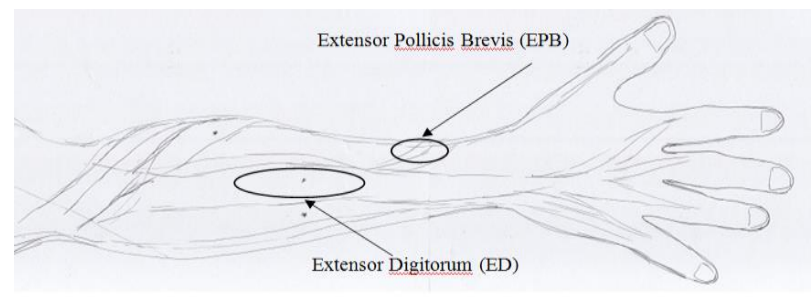

Figure 5. Sketch for the posterior view of a human forearm with the location of muscles under consideration: EPB and ED

\section{B. EMG Data Collection}

The EMG data collection was carried out for six classes of forearm movement summarized as: hand open (HO), hand closed (HC), thumb-index finger pinch (FP1), thumb-middle finger pinch (FP2), thumb-ring finger pinch (FP3), and thumb-little finger pinch (FP4). When collecting the data, the subjects were instructed to pinch/grasp the hand dynamometer 
at maximum strength which were recorded and considered as subject's maximum voluntary contraction (MVC). Then, they were asked to produce each of the six specific movements at different finger pinch and hand grip strength $(20,40,60,80$, and $100 \%$ of MVC), and hold that movement for a period of $10 \mathrm{sec}$ with $5 \mathrm{sec}$ rest sessions incorporated in between each movement for three trials. The experiments were repeated three times to prepare datasets that are required for the mapping process; two sets were used as training data while one set was used as testing data for the neural network.

In general, the estimation of muscle excitation can be done by analyzing the amplitude of the EMG signals generated. More motor units and higher firing rates are needed in order to maintain or increase the pinching/gripping forces. The quality and accuracy of the EMG signals collected were improved by ensuring accurate electrodes placement for each muscle under consideration for specific movement. The subjects were instructed to perform several tests to detect the right location of each muscle as described based on [21] and are listed as in Table 2.

TABLE II. MUSCLES IDENTIFICATION TEST AND ITS LOCATION FOR ELECTRODE PLACEMENT

\begin{tabular}{|l|l|l|l|}
\hline No & \multicolumn{1}{|c|}{ Muscle } & \multicolumn{1}{|c|}{ Test } & \multicolumn{1}{c|}{ Location } \\
\hline 1 & $\begin{array}{l}\text { Flexor } \\
\text { Digitorum } \\
\text { Superficialis } \\
\text { (FDS) }\end{array}$ & $\begin{array}{l}\text { Forearm in supination, one } \\
\text { finger is flexed at the PIP joint, } \\
\text { while the DIP are kept } \\
\text { extended against resistance } \\
\text { and the other three fingers are } \\
\text { held in an extended to } \\
\text { inactivate the FDP. }\end{array}$ & $\begin{array}{l}\text { Middle of the } \\
\text { forearm, index } \\
\text { fingers flexed } \\
\text { towards biceps } \\
\text { tendon, just } \\
\text { medial to the } \\
\text { finger. }\end{array}$ \\
\hline 2 & $\begin{array}{l}\text { Flexor } \\
\text { Digitorum } \\
\text { Profundus } \\
\text { (FDP) }\end{array}$ & $\begin{array}{l}\text { Forearm in supination, the PIP } \\
\text { joint and middle phalanx are } \\
\text { held in the extended position } \\
\text { while the person attempts to } \\
\text { flex the DIP joint against } \\
\text { resistance. }\end{array}$ & $\begin{array}{l}\text { lower 1/3 of } \\
\text { forearm and 2 } \\
\text { fingerbreadth, } \\
\text { volar to the } \\
\text { ulna. }\end{array}$ \\
\hline 3 & $\begin{array}{l}\text { Extensor } \\
\text { Digitorum } \\
\text { (ED) }\end{array}$ & $\begin{array}{l}\text { Forearm in pronation, the } \\
\text { fingers are extended at MCP } \\
\text { joints while pressure are } \\
\text { exerted at the PIP joints by } \\
\text { attempting to flex them. }\end{array}$ & $\begin{array}{l}\text { Upper 1/3 of } \\
\text { forearm } \\
\text { between radius } \\
\text { and ulna. }\end{array}$ \\
\hline 4 & $\begin{array}{l}\text { Flexor } \\
\text { Pollicis } \\
\text { Longus } \\
\text { (FPL) }\end{array}$ & $\begin{array}{l}\text { Forearm in supination, the } \\
\text { proximal phalanx of the thumb } \\
\text { is held and distal phalanx is } \\
\text { flexed against resistance. }\end{array}$ & $\begin{array}{l}\text { Middle of the } \\
\text { forearm, volar } \\
\text { to the radius. }\end{array}$ \\
\hline 5 & $\begin{array}{l}\text { Extensor } \\
\text { Pollicis } \\
\text { Brevis }\end{array}$ & $\begin{array}{l}\text { Forearm in pronation, the } \\
\text { thumb is extended at the MCP } \\
\text { joint against resistance. }\end{array}$ & $\begin{array}{l}\text { Lower 1/3 of } \\
\text { forearm } \\
\text { between radius } \\
\text { and ulna. }\end{array}$ \\
\hline
\end{tabular}

\section{PATTERN RECOGNITION PROCESS}

The structure of the EMG based control method can be categorized as pattern-recognition or non-pattern recognition based. Non-pattern recognition normally consists of a simple structure with only few processing steps. It is normally addressed as the primary level controller with ON/OFF function. It is practically not adequate to control exoskeleton hand as its accuracy is normally lower as compared to the pattern recognition based methods. Thus, pattern recognition based method was adopted in this research. The EMG signals were processed in four stages; EMG data acquisition, data segmentation, feature extraction and classification.
In the EMG data acquisition stage, the raw EMG signals were collected with sampling frequency of $2 \mathrm{kHz}$ obeying the Nyquist theorem which suggests that the sampling frequency should be at least twice the highest frequency contained in the signal. In general, the usable energy of the surface EMG signal is limited to the 0 to $500 \mathrm{~Hz}$ frequency range, with the dominant energy being in the $50-150 \mathrm{~Hz}$ range. Then, the data were filtered using second-order bandpass filter $(20 \mathrm{~Hz}-450$ $\mathrm{Hz}$ ) in MATLAB environment. The data segmentation was performed using overlapping segmentation technique with $256 \mathrm{~ms}$ window size and $128 \mathrm{~ms}$ window increment. This method has advantages over disjoint segmentation as it increased processing time and provided better classification performance.

Feature extraction method plays a critical role in extracting useful information hidden in the forearm EMG signals. In this research, the time-domain features; root mean square (RMS), mean absolute value (MAV), zero-crossing rate (ZC) and waveform length (WL) were chosen due to their computational simplicity.

RMS is widely adopted for feature extraction [22]. It is modelled as amplitude modulated Gaussian random process. It is related to the constant force and non-fatiguing contraction of the EMG signal [23]. It can be defined as:

$$
R M S=\sqrt{\frac{1}{N} \sum_{i=1}^{N} x_{i}{ }^{2}}
$$

MAV is the average of the absolute value of EMG signal measured for certain duration of time. It is an easy way for detection of muscle contraction levels and is one of the most popular used in EMG signal analysis. MAV feature can be defined as:

$$
M A V=\left(\frac{1}{N}\right) \sum_{i=1}^{N}\left|x_{i}\right|
$$

$\mathrm{ZC}$ is the number of times that EMG signal has crossed the zero line (from negative to positive or vice versa). Threshold condition is added to avoid background noises and is implemented as:

$$
\begin{aligned}
& Z C=\sum_{i=1}^{N-1}\left(\operatorname{sgn}\left(x_{i} \times x_{i+1}\right) \cap\left|x_{i}-x_{i+1}\right|\right) \geq \text { threshold } \\
& \operatorname{sgn}(x)= \begin{cases}1, & \text { if } x \geq \text { threshold } \\
0, & \text { otherwise }\end{cases}
\end{aligned}
$$

WL is the accumulative length of the EMG waveform over the time segment and can be defined as:

$$
W L=\sum_{i=1}^{N-1}\left|x_{i+1}-x_{i}\right|
$$

A two-layer neural network was employed as the pattern classifier to map the time-domain features into fingers joint angles. A feed-forward backpropagation algorithm was used to train the neural network. The input matrices (time-domain features) and corresponding target matrices (the fingers joint angles) were used to train the neural network until it can approximate a function by associating the elements in the input matrices with elements in the target matrices.

Separate neural networks were used to model each finger (the four medial fingers and the thumb). The neural network 
designed for the four medial fingers consist of 3-layers (an input layer with 3 neurons; features from FDS, FDP and EDC muscles at $40 \%$ finger pinch/hand grip strength), a tangentsigmoid hidden layer with 15 neurons and a pure linear output layer with 1 neuron (joint angles). As for the thumb, the designed neural network consists of 2-layers (an input layer with 2 neurons; features from FPL and EPB muscles at $40 \%$ finger pinch/hand grip strength), a tangent-sigmoid hidden layer with 10 neurons and a pure linear output layer with 1 neuron (joint angles). The results are discussed in the next session.

\section{EXPERIMENTAL RESULTS}

Results obtained at each stage of the research are presented in this section. The effectiveness of muscle selected and accuracy of raw EMG signal collected were evaluated by analysing the change in signal amplitude towards different finger pinch and hand grip strength. Figure 6 reveals that the amplitude increases proportionally to the increase in the hand grip strength. The raw EMG was collected at different hand grip strengths $(20,40,60$, and $80 \%$ of MVC) for FDS muscle at neutral wrist position. It is noted in the EMG trace that, the signal envelope of EMG activities shows many spikes indicating the brain activity to electrically activate/excite the muscle to produce the desired hand grip strength. In the case of fatigue, the envelope of the muscle will be increasing even though the force is actually decreasing indicating that the brain is driving the muscle harder and harder but the muscle fatigue makes it weaker.

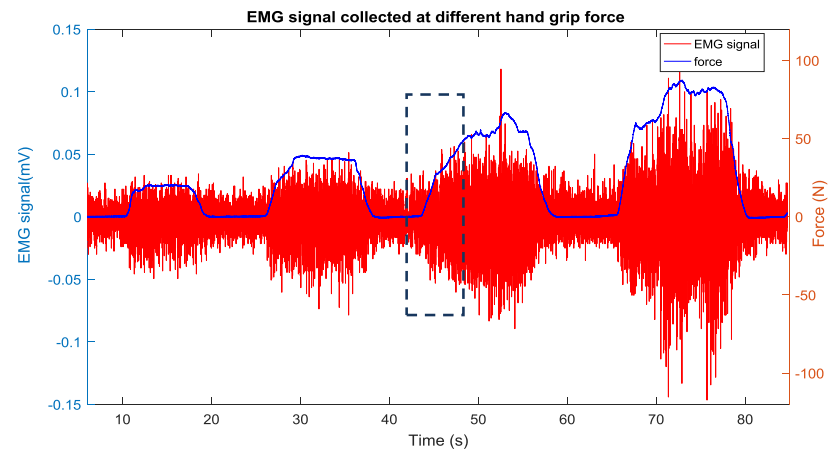

Figure 6. The raw EMG collected at different hand grip strength (20, 40, 60 , and $80 \%$ of MVC) for FDS muscle at neutral wrist position. It can be seen clearly from the EMG envelope that the muscle is electrically activated before the force generation as indicated by the dotted line.

In the second part of the experiments, the plot of the EMG features extracted from FDS and EDC muscles for four medial fingers were first observed to visually inspect the distribution of the extracted features across different finger pinch/hand grip movements as shown in Figure 7 to Figure 10. It is noted in these figures that separable classes of features with WL method produced more significant features when compared to RMS, MAV and ZC methods. In particular, it shows that WL features appear to be the best sensing technique to represent the intention of the user. Thus, the WL features were chosen in classifying the control input. Similar analysis was conducted and concluded for the thumb based on data collected at FPL and EPB muscles.

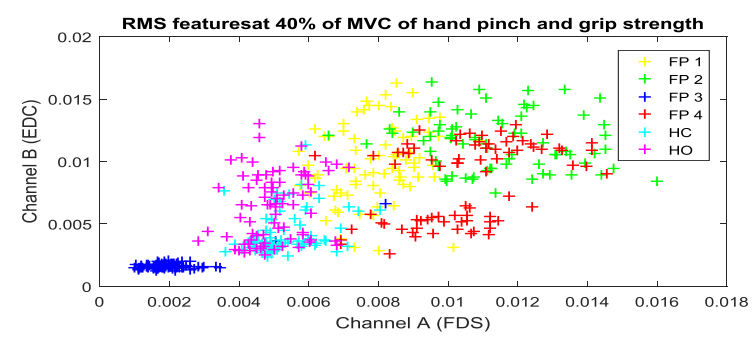

Figure 7. RMS features extracted (from FDS and EDC muscles) at $40 \%$ of MVC finger pinch strength and different hand movements (hand open and hand closed) for the four medial fingers.

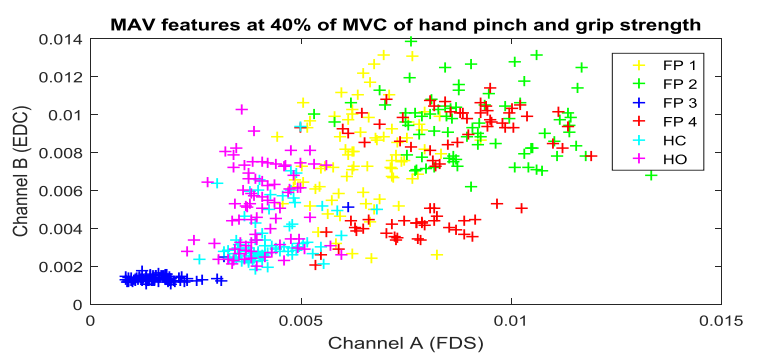

Figure 8. MAV features extracted (from FDS and EDC muscles) at $40 \%$ of MVC finger pinch strength and different hand movements (hand open and hand closed) for the four medial fingers.

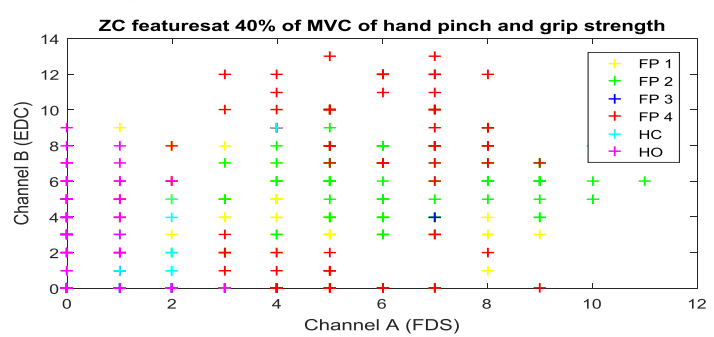

Figure 9. ZC features extracted (from FDS and EDC muscles) at $40 \%$ of MVC finger pinch strength and different hand movements (hand open and hand closed) for the four medial fingers.

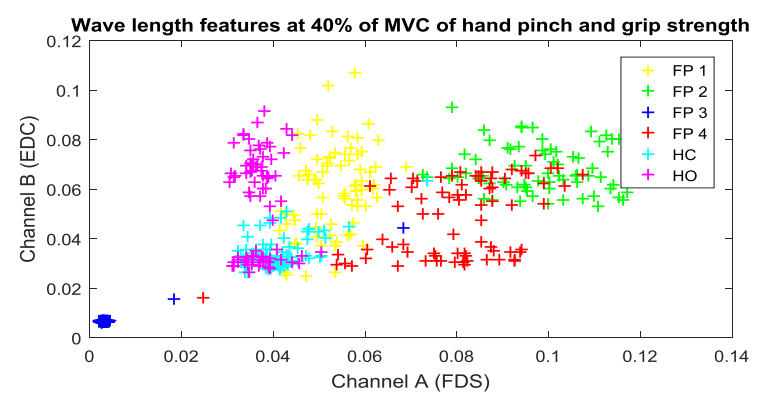

Figure 10. WL features extracted (from FDS and EDC muscles) at $40 \%$ of MVC finger pinch strength and different hand movements (hand open and hand closed) for the four medial fingers.

Several datasets comprising of WL features from flexion and extension muscles were then used as the training and testing data for classifying the control input. Figure 11 and Figure 12 show the input signals and the corresponding joint angles (MCP \& PIP) estimated by the neural network for the four medial fingers and the thumb. The performances of each of the neural networks are evaluated by calculating the Mean Absolute Error (MAE) between the output values estimated by the neural network and actual values. It can be concluded that the neural networks used managed to estimate the joint 
angles for all five fingers from the WL features with acceptable MAE values.
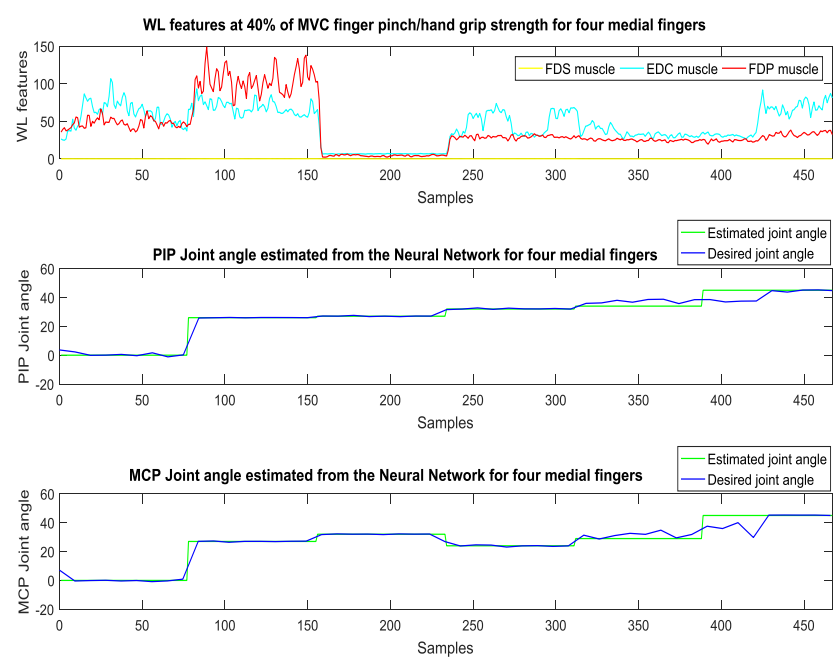

Figure 11. Joint angles predicted using neural networks when WL features from FDS, FDP and EDC muscles were used as the input signals.
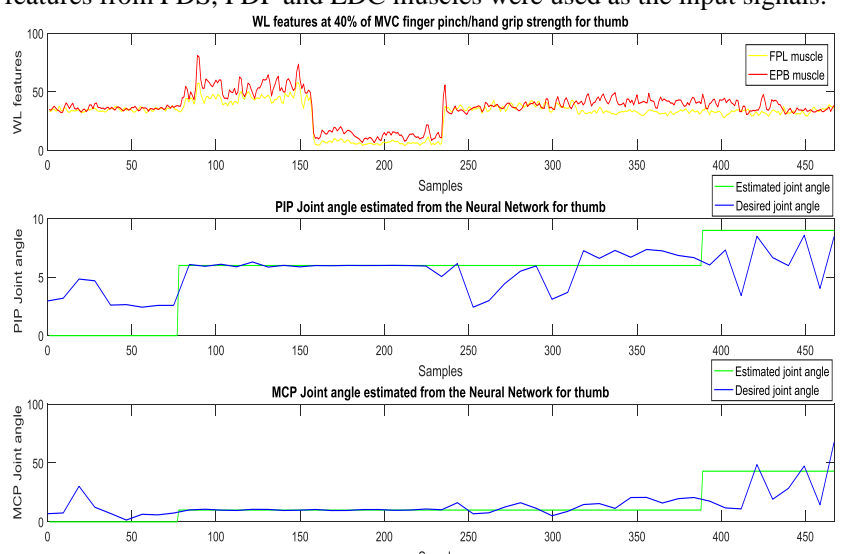

Figure 12. Joint angles predicted using neural networks when WL features from FPL and EPB muscles were used as the input signals.

\section{CONCLUSION AND FUTURE WORK}

As a conclusion, the features extracted from the datasets have significantly presented separable class and range for user defined input signals. The inter-relation between the EMG signal, various finger pinch/hand grip strengths and joint angles for five fingers have been established and can be used as control input to control the exoskeleton hand. Since the proposed work only discussed the myoelectric modeling for the fingers, the work should be extended for the wrist movement using similar methods. Besides, control framework that integrates all the fingers and wrist should also be designed to control the exoskeleton hand that can resemble movement and/or grasping abilities of the human hand.

\section{REFERENCES}

[1] S. Association, "State of the Nation Stroke statistics," ed, January 2017.

[2] S. Association, "Physical effects of stroke," ed, January 2013.

[3] R. A. R. C. Gopura, K. Kiguchi, and D. S. V. Bandara, "A brief review on upper extremity robotic exoskeleton systems," in 2011 6th international Conference on Industrial and Information Systems, 2011, pp. 346-351.
[4] R. C. a. L. C. J.L. Pons, Introduction to wearable robotics, in Wearable robots: Biomechatronic Exoskeletons, J. L. Pons, Ed., ed: John Wiley \& sons 1td, 2008.

[5] K. Kiguchi, "Active Exoskeleton for Upper-limb Motion Assist," International Journal of Humanoid Robotics, vol. 04, pp. 607-624, 2007.

[6] Y. Fan and Y. Yin, "Active and Progressive Exoskeleton Rehabilitation Using Multisource Information Fusion From EMG and Force-Position EPP," IEEE Transactions on Biomedical Engineering, vol. 60, pp. 3314-3321, 2013.

[7] P. Maciejasz, J. Eschweiler, K. Gerlach-Hahn, A. Jansen-Troy, and S. Leonhardt, "A survey on robotic devices for upper limb rehabilitation," Journal of NeuroEngineering and Rehabilitation, vol. 11, pp. 1-29, 2014.

[8] G. Gilardi, E. Haslam, V. Bundhoo, and E. J. Park, "A shape memory alloy based tendon-driven actuation system for biomimetic artificial fingers, part II: modelling and control," Robotica, vol. 28, pp. 675-687, 2010.

[9] M. D. Baker, M. K. McDonough, E. M. McMullin, M. Swift, and B. F. BuSha, "Orthotic Hand-Assistive Exoskeleton," IEEE 37th Annual Northeast Bioengineering Conference (NEBEC), 2011.

[10] M. F. Rotella, K. E. Reuther, C. L. Hofmann, E. B. Hage, and B. F BuSha, "An orthotic hand-assistive exoskeleton for actuated pinch and grasp," in IEEE 35th Annual Northeast Bioengineering Conference, 2009, pp. 1-2.

[11] L. A. Martinez, O. O. Olaloye, M. V. Talarico, S. M. Shah, R. J. Arends, and B. F. BuSha, "A power-assisted exoskeleton optimized for pinching and grasping motions," in IEEE 36th Annual Northeast Bioengineering Conference (NEBEC), 2010, pp. 1-2.

[12] B. L. Shields, Main, J. A., Peterson, S. W. and Strauss, A. M., "An anthropomorphic hand exoskeleton to prevent astronauthand fatigue during extravehicular activities", in IEEE Transactions on Systems, Man and Cybernetics, Part A: Systems and Humans, 1997.

[13] J. Yang, H. Xie, and J. Shi, "A novel motion-coupling design for a jointless tendon-driven finger exoskeleton for rehabilitation," Mechanism and Machine Theory, vol. 99, pp. 83-102, 5// 2016.

[14] N. S. K. Ho, K. Y. Tong, X. L. Hu, K. L. Fung, X. J. Wei, W. Rong, et al., "An EMG-driven exoskeleton hand robotic training device on chronic stroke subjects: Task training system for stroke rehabilitation," in IEEE International Conference on Rehabilitation Robotics, 2011, pp. 1-5.

[15] B. Cesqui, P. Tropea, S. Micera, and H. I. Krebs, "EMG-based pattern recognition approach in post stroke robot-aided rehabilitation: a feasibility study," Journal of NeuroEngineering and Rehabilitation, vol. 10, pp. 1-15, 2013

[16] E. B. Brokaw, I. Black, R. J. Holley, and P. S. Lum, "Hand Spring Operated Movement Enhancer (HandSOME): A Portable, Passive Hand Exoskeleton for Stroke Rehabilitation," IEEE Transactions on Neural Systems and Rehabilitation Engineering, vol. 19, pp. 391-399, 2011.

[17] Y. Hasegawa, J. Tokita, K. Kamibayashi, and Y. Sankai, "Evaluation of fingertip force accuracy in different support conditions of exoskeleton," in Robotics and Automation (ICRA), IEEE International Conference, 2011, pp. 680-685.

[18] H. Yasuhisa, M. Yasuyuki, W. Kosuke, and S. Yoshiyuki, "Fivefingered assistive hand with mechanical compliance of human finger," in Robotics and Automation, IEEE International Conference, 2008, pp 718-724.

[19] W. Yu, J. Rosen, and X. Li, "PID admittance control for an upper limb exoskeleton," in Proceedings of the 2011 American Control Conference, 2011, pp. 1124-1129.

[20] K. S. Lee and M. C. Jung, "Flexion and Extension Angles of Resting Fingers and Wrist", International Journal of Occupational Safety and Ergonomics, 2015.

[21] K. L. Moore, and A. F. Dalley, Clinically Oriented Anatomy. Philadelphia: Lippincott Williams \& Wilkins, 1999, ch. 6.

[22] S. W. H. M. T. D. Lalitharatne, "A Study of Controlling UpperLimb Exoskeletons Using EMG and EEG signals " Ph.D. Dissertation, Department of Science and Advanced Technology,, Saga University, 2014

[23] A. Phinyomark, P. Phukpattaranont, and C. Limsakul, "Feature reduction and selection for EMG signal classification", $A n$ International Journal of Expert Systems with Applications, 2012, pp $7420-7431$. 